Pla-Campas, G., Arumí-Prat, J. \& Simó-Gil, N. (2022). Análisis de los efectos de un proyecto innovador de codocencia en la universidad. Revista Electrónica Interuniversitaria de Formación del Profesorado, 25(1), 93-104.

\title{
Análisis de los efectos de un proyecto innovador de co-docencia en la universidad
}

\author{
Gil Pla-Campas, Joan Arumí-Prat, Núria Simó-Gil \\ Universitat de Vic - Universitat Central de Catalunya
}

\section{Resumen}

Con la voluntad de mejorar la enseñanza en la universidad se presenta el estudio de un proyecto de innovación didáctica en la educación superior que se corresponde con el modelo de codocencia de creación de equipo. El proyecto implica la integración de dos asignaturas con profesores, objetivos, cargas formativas e incluso tipos de aulas diferentes. El trabajo pretende mostrar los efectos de la aplicación de un modelo de docencia no convencional en la experiencia académica del alumnado y del profesorado. Mediante un enfoque cualitativo y a través de cuestionarios, grupos de debate y entrevistas en profundidad se analizan los efectos de la codocencia en tres categorías de análisis distintas: la logística del programa, el aprendizaje del alumnado y el rol del profesorado. Los resultados indican que los efectos son de magnitud diferente en cada dimensión de análisis y, además, que los efectos en estas dimensiones se perciben de manera distinta según los agentes. A modo de conclusiones, el trabajo concluye los tres efectos más significativos para iniciar y mantener proyectos de codocencia en educación superior.

\section{Palabras clave}

Co-docencia; educación superior; equipo docente; estrategias docentes.

\section{Contacto:}

Gil Pla-Campas, gil.pla@uvic.cat, Facultat d’Educació, Traducció, Esports i Psicologia, c/ Sagrada Família nº 708500 - Vic, Barcelona. 


\title{
Analysis of the effects of an innovative co-teaching project in Higher Education
}

\begin{abstract}
A study of a didactic innovation project in higher education based on the team-building co-teaching model is presented. The project involves the integration of two subjects with two teachers, different objectives, training loads and even different types of classrooms. The purpose of this research is to show the effects of the application of this unconventional teaching model in the academic experience of students and teachers. Through a qualitative approach and by means as of questionnaires, discussion groups and in-depth interviews; the research studies the effects of teaching experience in three major categories of analysis: the logistics of the program, student learning and the role of teachers. The results show different magnitude of the effects for each dimension of analysis and, in addition, results also show different perceptions for each dimension of analysis in accordance with each agents' perspective. As a conclusion, the work concludes the three most significant effects for starting and maintaining co-teaching projects in higher education.
\end{abstract}

\section{Key words}

Coteaching; Higher Education; Team Teaching; Teaching Strategies.

\section{Introducción}

Este artículo se enmarca en un proyecto de desarrollo de prácticas de innovación docente en la educación superior, en concreto, en la aplicación de una propuesta de codocencia en la Universidad. Para Flecha y otros (2014) la codocencia es una de las actuaciones educativas de éxito en el contexto universitario y se trata de la impartición de asignaturas por parte de dos profesores con orientaciones teóricas diferentes. Un profesor plantea el tema desde una perspectiva y, al mismo tiempo, en la misma clase, el otro profesor/a plantea una perspectiva diferente, siempre con argumentos basados en evidencias. La codocencia pretende un diálogo igualitario entre diferentes perspectivas que comporta beneficios, pero también retos, para los docentes y los estudiantes (Flecha y otros, 2014).

La codocencia se inició como un enfoque instructivo para apoyar a los estudiantes con discapacidad en las aulas de educación general (Hang y Rabren, 2009) y posteriormente se ha erigido como un enfoque para la inclusión educativa en el que un docente y un especialista trabajan conjuntamente (Friend y otros, 2010). En el ámbito de la educación superior hay numerosas investigaciones en la formación del profesorado que coinciden con las posibles ventajas de dicha estrategia pedagógica (Bouck, 2007; Cohen y DeLois, 2002; Duran-Gisbert y otros, 2019; Graziano y Navarrete, 2012). Algunas de estas prácticas pretenden desarrollar prácticas de innovación educativa tal como han analizado Castro Zubizarreta y otros (2017) o Pincheira Retamal y Otondo Briceño (2016).

Suárez-Díaz (2016) afirma que los tres componentes básicos de la codocencia: la coplanificación, la coinstrucción y la coevaluación permiten a los profesionales que comparten instrucción, combinar sus conocimientos y habilidades para crear ambientes formativos en los que la enseñanza sea rigurosa y flexible, es decir basada en estándares y a su vez adaptada a las necesidades de aprendizaje de cada estudiante (Friend y otros, 2010). La codocencia favorece la generación de un marco de retroalimentación constante para el profesorado implicado, al compartir su experiencia y conocimientos durante el desarrollo de las sesiones (Rodríguez, 2014). Además, la enseñanza en contexto mejora el grado de relación que los estudiantes sienten con sus iguales y docentes (Fedesco y otros, 2020). 
Por ello, la coordinación, el consenso de objetivos, la alternancia de roles y la interdependencia son relevantes en el desarrollo de prácticas de codocencia (Castro Zubizarreta y otros, 2017). El interés sobre esta práctica orienta la investigación de la codocencia hacia tres temas clave: a) las cuestiones relacionadas con la logística del programa, incluyendo la planificación común del profesorado y la programación de los estudiantes en clases compartidas; b) el impacto de la codocencia en el aprendizaje, el comportamiento y las percepciones de los estudiantes, incluyendo los informes sobre logro académico, asistencia y disciplina; y c) los roles y las relaciones de los profesores, incluidas sus percepciones sobre la codocencia y su impacto y eficacia (Friend y otros, 2010). En esta línea cabe preguntarse si la codocencia en educación superior puede contribuir a que los estudiantes alcancen aprendizajes de calidad competencial desarrollando habilidades complejas como: la contextualización de la información, la posibilidad de establecer relaciones entre contenidos, emitir valoraciones al respecto y aplicar dicha información a diversas situaciones (Torres Soto y Vallejo Ruiz, 2018).

Morelock y otros (2017) diferencian dos tendencias en codocencia, la primera plantea una alternancia de responsabilidades entre los dos profesores implicados, mientras que la segunda propone la enseñanza de forma conjunta. Uno de los retos de la codocencia es el desarrollo de colaboraciones entre el profesorado que construyan una comunidad de aprendizaje (Guise y otros, 2017) y sea posible aprender del proceso docente (Guise y otros, 2021) que revaloriza tanto el conocimiento como la experiencia (Tardif, 2004). La investigación de Härkki y otros (2021) destaca la codocencia como una práctica colaborativa en la que se evidencia que, además de compartir la planificación, la instrucción y la evaluación, las prácticas de codocencia de mayor calidad comparten un debate sobre el enfoque del contenido que los docentes enseñan; el profesorado colabora en contextos integrados de la actividad docente; es clave la retroalimentación y la reflexión entre docentes; y las prácticas gozan de una duración sostenida (Darling-Hammond, Hyler y Gardner, 2017). Así mismo contar con suficientes recursos físicos y humanos y con el apoyo de comunidades activas orientadas al desarrollo de práctica son condiciones que mejoran las prácticas de codocencia. Por todo ello, el contexto de realización es fundamental para el éxito de la codocencia.

Tal y como sugieren Dieker y Murawski (2003), la codocencia se fundamenta en la equiparación de los roles docentes y en la docencia simultánea, por eso, la codocencia no permanece estática y la disponibilidad al cambio se muestra como un beneficio de la docencia compartida (Yoo y otros, 2019). Considerando el contexto de colaboración y apoyo mutuo en el que se desarrolla la codocencia, los mismos autores observan que la experiencia fomenta la creatividad del profesorado involucrado, fomenta su capacidad de reflexionar sobre la práctica docente $y$, en definitiva, viven el contexto de docencia compartida como una práctica orientada al desarrollo profesional. En este sentido, la codocencia ofrece un marco de análisis sobre la práctica a partir de la conversación reflexiva (Crow y Smith, 2005). De todos modos, iniciarse con estrategias de codocencia exige unas condiciones organizativas mínimas que sin ellas no es posible avanzar hacia cambios educativos de mayor calado. Chanmugam y Gerlach (2013) definen cuatro condiciones: a) el apoyo institucional; b) la aclaración de los roles de comunicación y reparto de responsabilidades entre ambos profesores; c) una conceptualización de los contenidos durante el curso y d) un plan inicial.

Así pues, la finalidad de nuestra investigación se centra en el análisis de dichas condiciones para iniciar y mantener de forma sostenida prácticas de codocencia en la educación superior. Concretamente el artículo tiene el objetivo de dar a conocer los efectos de la codocencia en la logística, el aprendizaje de los alumnos y el rol de los docentes en un proyecto de creación 
de un equipo universitario a través de la experiencia académica vivida por el alumnado y el profesorado.

\section{Metodología}

Para observar los efectos de la codocencia en la experiencia vivida por el alumnado y el profesorado en la creación de un equipo docente, se propone una investigación con un diseño mixto, de carácter descriptivo y no experimental.

\section{Participantes}

Por las características de la propuesta, se utiliza una muestra no probabilística y por conveniencia conformada por 120 sujetos estudiantes de $1 \mathrm{r}$ semestre de $1 \mathrm{r}$ curso del grado de Ciencias de la Actividad Física y el Deporte (CAFD). En el momento de iniciar el curso, el grupo está compuesto por 100 hombres y 20 mujeres de entre 17 y 25 años $(M=19,82$ y $D S=2,08)$.

\section{Procedimiento e intervención}

La intervención ha sido llevada a cabo durante el 1er semestre de 1er curso del grado de CAFD con dos asignaturas de disciplinas distintas. Las asignaturas son Aprendizaje y Desarrollo Motor (ADM), de 6 créditos ECTS y Deporte Colectivo I: Baloncesto (Baloncesto) de 4,5 créditos ECTS. Si, por un lado, ADM es una asignatura de formación básica y conceptual del ámbito de la psicología, Baloncesto es una asignatura de carácter eminentemente práctico y especializada del grado.

La implementación del proyecto implicó diversas modificaciones didácticas en tres niveles sistémicos y temporales de actuación. Un nivel macro e institucional iniciado el curso anterior al proyecto piloto. Un segundo nivel meso y curricular iniciado también el curso anterior a la implementación, pero igualmente vivo durante el desarrollo del proyecto. $Y$ un nivel micro de intervención en el aula atento a las relaciones pedagógicas (profesor-alumno, equipo docente y alumnos-alumnos) de la experiencia. La siguiente figura muestra los niveles y las modificaciones básicas realizadas:

Figura 1.

Niveles de modificación de la propuesta codocencia
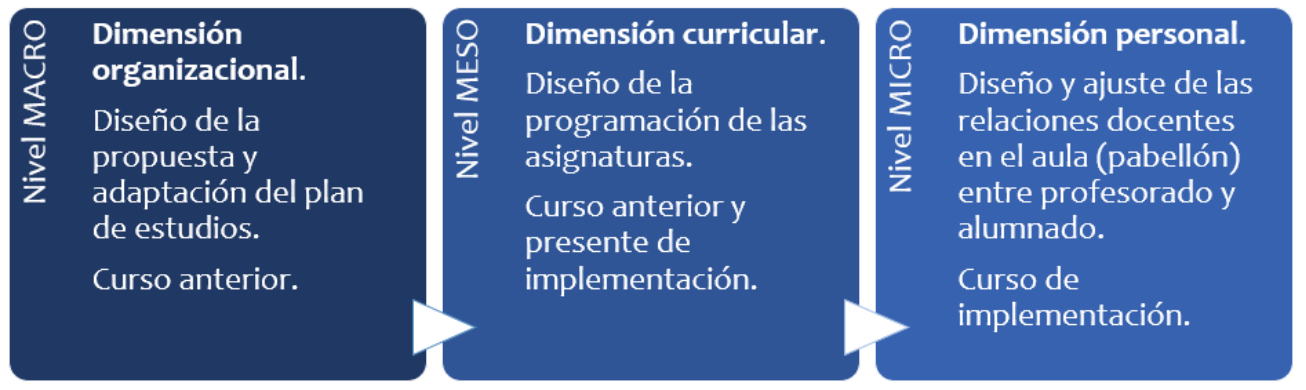

Para favorecer la creación del equipo docente se debía hacer modificaciones en el nivel macro como: cambio de semestre de una asignatura, selección de las franjas docentes compartidas y elaboración de nuevos horarios de curso para el alumnado. Estos cambios permitieron que las dos asignaturas pudieran compartir un bloque de sesión con el fin de realizar la codocencia (clases en un mismo espacio y tiempo). Concretamente se optó por 
compartir las franjas de clase de seminario, que es clase práctica, de Baloncesto y de seminario de ADM.

El nivel meso de las modificaciones permitió que, mientras el alumnado de Baloncesto aprendía este deporte, un grupo reducido de estudiantes de ADM (unos 25-30) observaba y analizaba las sesiones. Inmediatamente después, la situación se invertía: el grupo A realizaba $A D M$ y el grupo $B$, Baloncesto. El encadenamiento y alternancia de una asignatura con la otra permitió que, durante 3 horas consecutivas, el alumnado relacionara las dos asignaturas. Esta estructura se llevó a cabo 6 de los bloques de clase compartida de las 14 semanas del semestre e implicó el diseño de actividades vinculadas al contexto. En la misma línea, se adaptó y reorganizó la aparición de los contenidos en ambas asignaturas y se compartió materiales para el trabajo de ambas asignaturas.

Finalmente, el nivel de modificaciones micro implicó el ajuste del profesorado y alumnado a la nueva estructura didácticas. Desde la adaptación de un grupo de estudiantes a ser observado, a otro que observa; la gestión de la comunicación en un contexto no convencional como un pabellón, o la interacción entre grupos (observado-observador). La novedad del contexto, pues, implicó una atención específica a las dificultades del alumnado con el espacio, con la nueva dinámica de las sesiones, e incluso con las nuevas relaciones pedagógicas que se establecen con los dos profesores, y con el alumnado del otro grupo.

\section{Instrumentos de recogida de información}

Para observar los efectos de esta intervención, se utiliza tres instrumentos de recogida de información. Se implementa los tres instrumentos en la $13^{\text {a }}$ semana docente tras la entrega de los trabajos de ambas asignaturas, pero antes de conocer las calificaciones finales. Los instrumentos son:

a) Cuestionario de valoración de la experiencia para conocer la percepción de los efectos de la experiencia en el aprendizaje de los estudiantes. Se realizó mediante una escala Likert con dos ejes: a) el proceso de aprendizaje del propio estudiante y b) el aprendizaje mediante la observación de compañeros. Para cada eje, el alumnado evalúa el efecto de la codocencia en los siguientes indicadores: la percepción de aprendizaje en cada asignatura, el interés hacia las asignaturas y el contexto docente en general, y el interés sobre aspectos profesionales relacionados con las temáticas de la codocencia.

b) Grupos de debate para reconocer, desde un enfoque cualitativo, como se valora la experiencia del proyecto en el alumnado. Se organizó una dinámica de debate de 60' en clase de ADM a partir de los mismos grupos de trabajo habituales. La dinámica implicó debatir y responder a preguntas abiertas como: ¿cuál es la experiencia vivida en este contexto docente no convencional? ¿crees que esta experiencia ha potenciado tu aprendizaje en las asignaturas? o ¿qué te ha parecido tener dos profesores en un mismo espacio de clase? Se recogen las conclusiones de la valoración de cada grupo mediante un formulario de Google Forms, con una participación total de 20 grupos y 267 comentarios, organizados en cuatro ejes de análisis: fortalezas, debilidades, amenazas y oportunidades del proyecto.

c) Entrevistas en profundidad a cada profesor y por separado, para recopilar las reflexiones sobre la experiencia de codocencia. Se diseña una entrevista de estructura abierta y no dirigida. En el guion de la entrevista se formula una pregunta sobre el motivo del proyecto y otras tantas abiertas relacionadas con las tres categorías de análisis: logística, relación entre los dos profesores y aprendizajes del alumnado. El cierre de la entrevista sirve para exponer posibles perspectivas de mejora de la experiencia. 


\section{Análisis de los datos}

Las categorías de análisis de los datos surgen de los tres elementos que Friend et al. (2010) identifican como dimensiones más relevantes y frecuentes en de análisis en un proyecto de codocencia. Son las siguientes:

a) Logística del programa. Dicha categoría incluye la dimensión organizacional y ciertos aspectos de la dimensión curricular del proyecto y se ha recogido información de los grupos de debate y en las entrevistas en profundidad al profesorado.

b) Impacto en el aprendizaje del alumnado. Esta segunda categoría describe los efectos del proyecto sobre la percepción de aprendizaje del alumnado. Se han recogido datos en el cuestionario y en los grupos de debate.

c) Roles y relación entre el profesorado. Por último, esta categoría permite describir qué efectos produce la docencia no convencional y compartida del proyecto sobre cada profesor en sí mismo y sobre la interacción en el contexto.

\section{Resultados}

A continuación, exponemos los resultados según las tres categorías de análisis.

\section{Logística del programa}

El análisis de la percepción de los estudiantes con relación a la logística del programa se obtiene principalmente de los grupos de debate. El tema más destacado con más de la mitad de los comentarios en esta categoría es el del espacio de aprendizaje. Son 12 los comentarios de los grupos que hacen referencia al espacio inadecuado de la experiencia (las gradas de un pabellón deportivo) y 13 comentarios exponen que, el espacio provoca distracciones y falta de concentración en el alumnado. En contraposición, 9 comentarios de los grupos valoran positivamente el espacio porque les permite realizar la práctica deportiva y observar a los compañeros y compañeras llevando a cabo la práctica.

Con respeto a la logística del programa los profesores comentan en la entrevista otros aspectos sobre el espacio. En un primer momento, los profesores valoran el esfuerzo de coordinación del programa, pero no manifiestan en ningún momento de la entrevista los problemas que comporta el espacio para los estudiantes. El profesor de ADM comenta que lo más gratificante de la experiencia es "compartir un espacio de aprendizaje con un compañero porqué pone en juego tu propio saber de manera constante". El profesor de Baloncesto expone que "hay limitaciones logísticas de horario, de espacios y de plan de estudios en la universidad para llevar a cabo experiencias de codocencia". Los dos profesores aseguran en la entrevista estar satisfechos con la experiencia y con la posibilidad de romper esquemas y horarios que frecuentemente limitan propuestas innovadoras en la universidad.

\section{Impacto en el aprendizaje del alumnado}

Los tres instrumentos de recogida de datos proporcionan información sobre la percepción del efecto de la codocencia en el aprendizaje del alumnado. La estructura docente de compartir clase ha impactado positivamente y de forma relevante en la percepción de aprendizaje de los contenidos, en el interés de las asignaturas, en el interés de aspectos profesionales e incluso en las relaciones interpersonales ya que el contexto facilita el interés del alumnado hacia los miembros del otro grupo. En general, el grado de acuerdo de las afirmaciones es mayoritariamente alto o muy alto como muestra la tabla 1: 
Figura 2.

Resultados del cuestionario sobre la experiencia de aprendizaje. Compartiendo aula

\section{Compartir espacio y tiempo de clase entre ADM y Baloncesto}

...ha estimulado el interés de otras...

...ha incrementado mi interés por areas..

...ha incrementado mi interés por asistir..

...ha incrementado mi interés por..

...ha incrementado mi interés por ADM

...ha incrementado mi aprendizaje en.

...ha incrementado mi aprendizaje en ADM

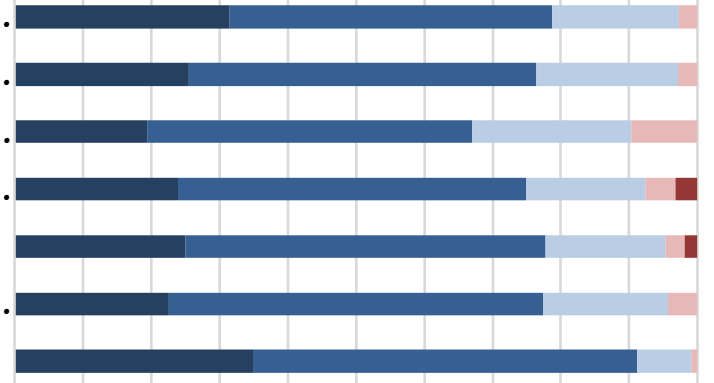

$\begin{array}{llllllllllll}0 & 0 & 10 \% & 20 \% & 30 \% & 40 \% & 50 \% & 60 \% & 70 \% & 80 \% & 90 \% & 100 \%\end{array}$

- Totalmente de acuerdo $\quad$ De acuerdo

$\square$ Ni de acuerdo ni en desacuerdo $\square$ En desacuerdo

- Totalmente de acuerdo

Además, los datos reflejan pequeñas diferencias en función de la asignatura ya que los efectos en ADM parecen ser valorados con más intensidad que en Baloncesto.

En una línea similar observamos de la entrevista que el profesor de ADM comenta que su alumnado estaba mejor acompañado en los conceptos, y específicamente considera que el alumnado había mejorado "la capacidad de comprensión de los saberes de la asignatura" y que tenía una "mejor capacidad interpretativa" para conectar teoría y práctica, aspecto central de sus aprendizajes. Con relación a los aprendizajes de Baloncesto, es decir aquellos vinculados al saber jugar, su profesor afirma que "no tengo evidencias de estos aprendizajes" asumiendo que una mejor comprensión del contexto no implica por ende un incremento de las habilidades del juego. La naturaleza distinta de los aprendizajes a los que el profesorado se refería, corporales de Baloncesto y analíticos de ADM, evita que las afirmaciones del profesorado sean contradictorias ya que se refieren a aprendizajes distintos. De hecho, el mismo profesor considera que "podríamos decir que los alumnos entendían mejor lo que practicaban porque lo hacían en la pista y lo miraban a sus compañeros desde la grada", aspecto que se refiere, este sí, a la vinculación teórico-práctica del deporte del Baloncesto.

En los grupos de debate el mayor número de comentarios, un total 128, son con relación a la categoría del impacto del aprendizaje en el alumnado. Si en el cuestionario de valoración los estudiantes afirman en más de un $80 \%$ que están de acuerdo y totalmente de acuerdo que la experiencia estimula las relaciones interpersonales, en los grupos de debate se reafirma este indicador. Los alumnos afirman en 18 comentarios que el hecho de trabajar en equipos reducidos fortalece su aprendizaje porqué fomenta la comunicación con los compañeros, la ayuda mutua y la comprensión de la información. 29 expresan la facilidad de conectar la teoría con la práctica, 6 citan la importancia de los comentarios del profesor en la clase y otros 9 muestran el valor del vídeo que realizaron como ayuda al aprendizaje. Cabe señalar que el trabajo en equipo también presenta desventajas tal y como recogen los 11 comentarios de los grupos de debate que se refieren al tiempo de espera cuando el profesor atiende a los equipos por turnos, o los 13 comentarios que hacen referencia a posibles mejoras en la dinámica del trabajo en equipo de los grupos. 
Los resultados en los cuestionarios indican que la experiencia favorece positivamente el interés de los alumnos por las asignaturas. En los grupos de debate también se aprecia esta valoración positiva en la relación entre los contenidos teóricos y prácticos. Un total de 13 comentarios señalan la oportunidad que es observar entrenamientos en directo. Este hecho permite a los estudiantes, tal y como se recoge en 16 comentarios, analizar distintos casos reales y entender mejor los contenidos teóricos de la asignatura de ADM. Aun así, hay propuestas de mejora en la experiencia como los 7 comentarios que hacen referencia a la necesidad de dar más autonomía a los estudiantes en el momento de entrar en la pista de baloncesto o distintos comentarios con relación a la importancia de la reflexión final del profesor de ADM.

En cuanto a la percepción del alumnado con relación a la estructura docente de dos asignaturas y dos profesores en el mismo momento, los resultados tienen una distribución similar puesto que, por un lado, el alumnado responde que es una experiencia formativa valorada positivamente en todos los sentidos (aprendizaje, interés temático, conexión profesional y relaciones interpersonales). La siguiente tabla muestra estos resultados:

Figura 3.

Resultados del cuestionario sobre la experiencia de aprendizaje. Observando compañeros

\section{Observar compañeros de clase...}

...ha estimulado el interés de otras..

...ha incrementado mi interés por areas..

...ha incrementado mi interés por asistir..

...ha incrementado mi interés por..

...ha incrementado mi interés por ADM

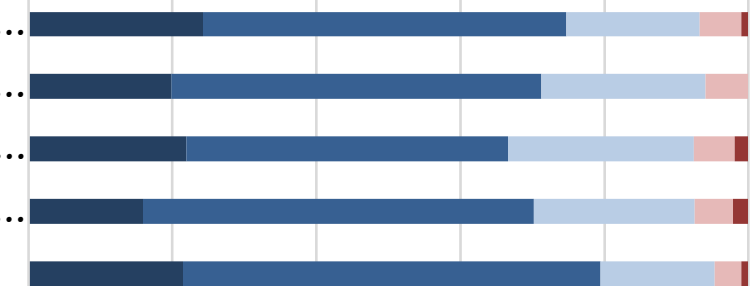

...ha incrementado mi aprendizaje en...

...ha incrementado mi aprendizaje en..

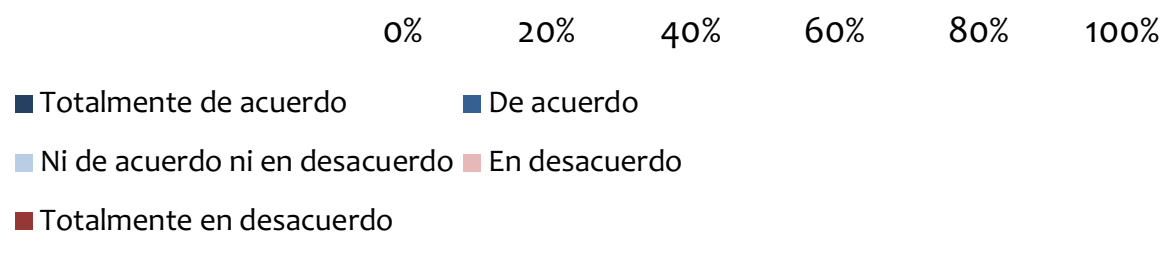

En general, las respuestas coinciden con el hecho de que compartir clase con colegas desconocidos no impide una experiencia de aprendizaje positiva. En consecuencia, se mantienen los valores del primer grupo de afirmaciones. Igualmente, se recoge valoraciones más positivas en la asignatura de ADM.

\section{Roles y relación entre los profesores}

Los datos de esta categoría se recogen principalmente con la entrevista a los dos profesores protagonistas de la experiencia. Aun habiendo demandas y oportunidades docentes diferentes entre el profesorado, los dos profesores aseguran que, a través de la experiencia, han fortalecido su relación. El profesor de ADM expone que "con el programa de codocencia los dos profesores nos dimos cuenta de que discutiendo sobre pedagogía deportiva 
coincidimos a la vez que discrepamos sobre muchos temas y es a partir de ahí, que podemos crecer y aprender individualmente haciendo clases y, a la vez, enseñar mejor". Los dos profesores, aunque están de acuerdo con el fortalecimiento de su relación, también coinciden en que compartir espacio docente con un compañero genera ciertas dudas y temores. El profesor de ADM explica: “El profesorado está acostumbrado a tener un aula con sus alumnos. En esta estrategia didáctica compartes el espacio y los alumnos con otro profesor. Tienes que estar abierto a los argumentos del otro profesor y a poner en duda los tuyos." Finalmente, los dos profesores en la entrevista exponen que aprendieron con su compañero. Mientras el profesor de Baloncesto aprendió del profesor de ADM "una forma de enseñar diferente, tanto en la forma de relacionarse con los estudiantes como de pensar el contexto de enseñanza" el profesor de Baloncesto aprendió del de ADM "el hecho de saber defender tan bien y tan contundentemente cómo enseñar el deporte”.

En el instrumento de los grupos de debate se cuantifican 25 comentarios sobre los roles y la relación entre los profesores. De la totalidad de comentarios realizados en esta categoría 8 son positivos respecto a la buena comunicación entre profesores. También hay 8 comentarios negativos en relación con las discrepancias mostradas entre ellos y 2 comentarios que valoran positivamente estas discrepancias entre los puntos de vista de los profesores. Finalmente 7 comentarios hacen referencia a la buena actitud de los profesores a la hora de enseñar. Queremos señalar en esta categoría los 8 comentarios negativos respecto a las discrepancias mostradas entre ellos. Este dato indica que un porcentaje alto de grupos no alcanzó a comprender la discrepancia de los profesores en las clases como un elemento positivo que pretende fomentar un análisis más complejo de los contenidos explicados.

\section{Discusión}

La logística del programa se sitúa como un elemento clave en la percepción del éxito como indican Friend y otros (2010). Lo singular de la propuesta reside en la percepción diferenciada de los agentes. Es decir, mientras que el alumnado vive con intensidad y dificultad la logística, al cambiar sus hábitos de asistencia a clase y alterar las formas de comportamiento habituales; el profesorado considera que los condicionantes de la logística se superan sin que comporte una problemática específica como muestra la poca atención que dan a este tema en la entrevista. Posiblemente, el apoyo institucional de la coordinación del grado mostrado en el nivel meso de la propuesta, es clave para explicar esta reacción del profesorado. Estos resultados refuerzan la importancia de la dimensión organizacional de este tipo de proyectos en qué facilitar su logística, da seguridad al profesorado (Chanmugam y Gerlach, 2013).

En segundo lugar, los resultados sobre el efecto de la codocencia en el aprendizaje del alumnado permiten afirmar que hay indicios de aprendizajes relevantes. El análisis y triangulación de datos permite afirmar que los alumnos destacan aprendizajes en los que relacionan contenidos teóricos con los prácticos en la asignatura de ADM y manifiestan un mayor interés hacia el contexto formativo gracias al proyecto. Una percepción similar tiene el profesor de la asignatura de ADM quien afirma que el alumnado conecta con más facilidad ideas conceptuales con su expresión práctica. En este sentido, la estrategia de modificar el contexto docente, teniendo en cuenta los tres niveles, del micro al macro, mediante la experiencia inmersiva en contexto real (Fedesco y otros, 2020) impacta positivamente en la capacidad de integrar teoría con la práctica, la construcción del saber experiencial docente (Tardif, 2004).

En tercer lugar, los efectos de la experiencia impactan en la calidad del rol y en las relaciones del profesorado porque ha afectado en el saber experiencial docente que revaloriza tanto el conocimiento como la experiencia (Tardif, 2004) y en la creación de comunidad de 
aprendizaje (Guise y otros, 2017) gracias a las discusiones o contrastes entre ellos iniciadas en las clases. Cabe preguntarse por qué la discrepancia que es expresada como sensación de crecimiento personal por parte del profesorado, que nace de procesos de reflexión sobre la práctica (Crow y Smith, 2005), es percibida como una debilidad del proyecto por parte del alumnado. En futuras investigaciones será necesario analizar con más detalle cómo el profesorado especifica los roles de comunicación y reparto de responsabilidades (Chanmugam y Gerlach, 2013) y como afectan el aprendizaje del alumnado. El proyecto destaca al valor de la noción de equipo y su impacto positivo. Härkki y otros (2021) muestran la importancia de disponer de un profesorado comprometido con el proyecto, aspecto que se observa claramente por los orígenes y finalidades de este proyecto.

\section{Conclusiones}

La reflexión sistemática de esta propuesta y los resultados obtenidos muestran que una experiencia innovadora que modifique estructuras docentes convencionales y prestablecidas, como la propuesta, altera los ritmos docentes y de aprendizaje de sus agentes en la medida que cambia su proceder habitual. Los resultados muestran que el nuevo diseño docente conduce a cambio de hábitos e incertidumbre que dificultan el día a día. Solo si los gestores toman consciencia de ello, podrán actuar en consecuencia y gestionar mejor el contexto. Un contexto que, como hemos podido observar, se percibe de formas distintas según el agente observado. Así que, de este modo, la investigación apunta algunas de las oportunidades y los retos que surgen de esta propuesta para seguir mejorando la calidad educativa de la docencia universitaria.

Retomando la finalidad de nuestra investigación, destacamos los efectos más relevantes para iniciar y mantener prácticas de codocencia en la educación superior. Con respecto a la logística, la enseñanza en un contexto real necesita de unas condiciones adecuadas, así como de un acompañamiento docente que ayude al alumno a aprender de forma integrada. Con relación al aprendizaje del alumnado, la estructura codocente en contexto real fomenta la integración de aprendizajes teórico-prácticos de las asignaturas implicadas. Y finalmente, podemos afirmar que un proyecto de codocencia fortalece la relación entre el profesorado por que la convivencia en el aula estimula el debate pedagógico y el intercambio sobre los contenidos de enseñanza.

\section{Referencias}

Bouck, E. C. (2007). Co-Teaching... Not Just a Textbook Term: Implications for Practice. Preventing School Failure: Alternative Education for Children and Youth, 51(2), 46-51. https://doi.org/10.3200/psfl.51.2.46-51

Castro Zubizarreta, A., Briones Pérez, E. y Izquierdo Magaldi, B. (2017). La co-docencia en el contexto universitario como estrategia para la innovación docente. III Congreso Nacional de Innovación Educativa y Docencia En Red, 1-13. https://doi.org/10.4995/inred2017.2017.6721

Chanmugam, A. y Gerlach, B. (2013). A Co-Teaching Model for Developing Future Educators' Teaching Effectiveness. International Journal of Teaching and Learning in Higher Education, 25(1), 110-117.

Cohen, M. B. y DeLois, K. (2002). Training in tandem: Co-facilitation and role modeling in a group work course. Social Work with Groups, 24(1), 21-36. https://doi.org/10.1300/Joogv24n01_03 
Crow, J. y Smith, L. (2005). Co-teaching in higher education: reflective conversation on shared experience as continued professional development for lecturers and health and social care students. Reflective Practice, 6(4), 491-506. https://doi.org/10.1080/14623940500300582

Darling-Hammond, L., Hyler, M. E., Gardner, M. (2017). Effective Teacher Professional Development. Palo Alto.

Dieker, L. A. y Murawski, W. W. (2003). Co-Teaching at the Secondary Level: Unique Issues, Current Trends, and Suggestions for Success. The High School Journal, 86(4), 1-13. https://doi.org/10.1353/hsj.2003.0007

Duran-Gisbert, D., Flores-Coll, M., Mas-Torelló, O. y Sanahuja-Gavaldà, J.-M. (2019). Docencia compartida en la formación inicial del profesorado: potencialidades y dificultades según los estudiantes y los profesores. REIRE Revista d'Innovació i Recerca En Educació, 12(2), 1-11. https://doi.org/10.1344/reire2019.12.227430

Fedesco, H. N., Cavin, D. y Henares, R. (2020). Field-based Learning in Higher Education. Journal of the Scholarship of Teaching and Learning, 20(1), 65-84. https://doi.org/10.14434/josotl.v20i1.24877

Flecha, R., Racionero, S., Tintoré, M., y Arbós, A. (2014). Actuaciones de Éxito en la Universidad. Hacia la Excelencia Tomando las Mejores Universidades como Modelo. Multidisciplinary Journal of Educational Research, 4 (2), pp.131-150. doi: 10.4471/remie.2014.08

Friend, M., Cook, L., Hurley-Chamberlain, D. y Shamberger, C. (2010). Co-teaching: An illustration of the complexity of collaboration in special education. Journal of Educational and Psychological Consultation, 20(1), 9-27. https://doi.org/10.1080/10474410903535380

Graziano, K. J. y Navarrete, L. A. (2012). Co-Teaching in a Teacher Education Classroom: Collaboation, Compromise, and Creativity. Issues in Teacher Education, 21(1), 109-126.

Guise, M., Habib, M., Thiessen, K. \& Robbins, A. (2017). Continuum of co-teaching implementation: Moving from traditional student teaching to co-teaching. Teaching and Teacher Education, 66, 370-382. https://doi.org/10.1016/j.tate.2017.05.002

Guise, M., Hegg, S. y Robbins, A. (2021). Learning together through coteaching coaching: A model of support for coteaching pairs in clinical practice. Teaching and Teacher Education, 100, 103278. https://doi.org/10.1016/j.tate.2021.103278

Hang, Q. y Rabren, K. (2009). An examination of Co-teaching: Perspectives and efficacy indicators. Remedial and Special Education, 30(5), 259-268. https://doi.org/10.1177/0741932508321018

Härkki, T., Vartiainen, H., Seitamaa-Hakkarainen, P. y Hakkarainen, K. (2021). Co-teaching in non-linear projects: A contextualised model of co-teaching to support educational change. Teaching and Teacher Education, 97, 1-14. https://doi.org/10.1016/j.tate.2020.103188

Morelock, J. R., Lester, M. M. G., Klopfer, M. D., Jardon, A. M., Mullins, R. D., Nicholas, E. L. y Alfaydi, A. S. (2017). Power, perceptions, and relationships: A model of co-teaching in higher education. College Teaching, 65(4), 182-191. https://doi.org/10.1080/87567555.2017.1336610

Pincheira Retamal, J. y Otondo Briceño, M. (2016). Habilidades de co docencia en alumnos de Pedagogía en Educación Diferencial de la Universidad Católica de la Santísima Concepción. Revista de Estudios y Experiencias En Educación, 15(29), 95-108. https://doi.org/10.21703/rexe.201629951086

Rodríguez, F. (2014). La co-enseñanza, una estrategia para el mejoramiento educativo y la inclusión. Revista Latinoamericana de Educación Inclusiva, 8(2), 219-233.

Suárez-Díaz, G. (2016). Co-enseñanza: concepciones y prácticas en profesores de una Facultad de Educación en Perú. Revista Electrónica de Investigación Educativa, 18(1), 
$165-182$.

Tardif, M. (2004). Los saberes del docente y su desarrollo profesional. Narcea.

Torres Soto, A. y Vallejo Ruiz, M. (2018). ¿Contribuye la universidad al desarrollo de aprendizajes de calidad? Estudio descriptivo con estudiantes de la Universidad de Murcia. Revista Electrónica Interuniversitaria de Formación Del Profesorado, 21(1), 129. https://doi.org/10.6018/reifop.21.1.295261

Yoo, J., Heggart, K. y Burridge, N. (2019). Collaborative Coteaching (CCT): Practitioner Learning through Shared Praxis. Australian Journal of Teacher Education, 44(4), 6577. https://doi.org/10.14221/ajte.2018v44n4.5 